\title{
PPMS-based set-up for Raman and luminescence spectroscopy at high magnetic field, high pressure and low temperature
}

\author{
Matthias Hudl ${ }^{1,2^{*}+}$, Peter Lazor ${ }^{3 \dagger}$, Roland Mathieu ${ }^{4 \dagger}$, Alexander G Gavriliuk ${ }^{5,6+}$ and Viktor V Struzhkin ${ }^{7 \dagger}$
}

\footnotetext{
*Correspondence: hudl@kth.se

${ }^{\dagger}$ Equal contributors

${ }^{1}$ KTH Royal Institute of Technology, ICT Materials Physics, Electrum 229, SE-164 40 Kista, Sweden

2 Department of Materials, ETH Zürich, Vladimir-Prelog-Weg 4, $\mathrm{CH}-8093$ Zürich, Switzerland Full list of author information is available at the end of the article
}

\begin{abstract}
We present an experimental set-up permitting Raman and luminescence spectroscopy studies in a commercial Physical Properties Measurement System (PPMS) from

Quantum Design. Using this experimental set-up, gaseous, liquid and solid materials, in bulk or thin film form, may be investigated. The set-up is particularly suitable for the study of the spin-lattice coupling in strongly correlated oxide materials utilizing several different stimuli, e.g. magnetic and electric fields, high pressure and low temperatures. Details for the Raman extension, sample holder assembly and optical design, as well as data acquisition and measurement routine are described. Finally, we present exemplary results collected using the set-up, measured on reference materials, as well as on a correlated transition metal oxide.
\end{abstract}

Keywords: Raman spectroscopy; Quantum Design PPMS; High pressure; Ruby; Hydrogen conversion; Strongly correlated materials; Langasite

\section{Introduction}

Large efforts in the field of material science are spent on the development of novel functional materials. In this context, strongly correlated electron systems are a class of materials that promise a multitude of applications. Several transition metal oxides are being considered for the designing of new devices, owing e.g. to their magnetic, electric and dielectric [1-3] or optical and photovoltaic properties [4,5]. In such materials, the functionalities or physical properties are determined by the localization and delocalization of charge carriers [6]. As a result, magnetic, (di)electric, thermal, and optical properties are correlated with each other [6]. Inelastic light scattering from electronic charge density and phonons in correlated materials is a promising approach to evaluate such correlation properties [7]. This approach is deemed to be particularly fruitful if it can be done under the simultaneous influence of several stimuli that probe these properties such as, e.g. magnetic and/or electric fields, pressure and/or temperature.

Raman spectroscopy for instance is a versatile technique for studying structural properties, lattice distortions, and e.g. the coupling of spin and lattice degrees of freedom. This is due to different types of excitations that can be studied under the influence of external stimuli leading to observation of shifts in vibrational frequencies, changes in peak intensities and polarization states. This was successfully employed to study structure-property

(C) 2015 Hudl et al.; licensee Springer. This is an Open Access article distributed under the terms of the Creative Commons 
relationships in strongly correlated transition metal oxides [8,9]. A particular strength of electronic Raman scattering is the possibility to detect information on the electron dynamics from different regions of the Brillouin zone.

A Raman set-up for in-situ measurements of such properties at low temperature, magnetic field, electrical field and high pressure was constructed as an extension to the Quantum Design, Inc. Physical Properties Measurement System (PPMS) [10] - a standard cryogenic device in many laboratories world wide. An optical probe and an automatized data acquisition system were designed and linked with the already automatized temperature and magnetic field control provided by the PPMS. Several material properties can thus be investigated in the same PPMS set-up, using the standard set of options such as resistivity, magnetization or heat capacity, as well as using extensions permitting e.g. magneto(di)electric measurements [11] or the here proposed Raman spectroscopy setup. Dedicated custom-made devices for Raman spectroscopy with multiple excitations not using a Quantum Design PPMS were reported in literature previously $[12,13]$.

We present results collected on different reference systems, such as ruby and $\mathrm{H}_{2}$, as well as a transition metal oxide, $\mathrm{Ba}_{3} \mathrm{NbFe}_{3} \mathrm{Si}_{2} \mathrm{O}_{14}$, a langasite material with magnetically induced electronic polarization.

\section{Experimental apparatus and methods PPMS host system and Raman extension}

The Raman set-up is based on a commercial Quantum Design, Inc. PPMS [10] which incorporates (i) a cryostat providing a controllable variable temperature in the 2 - $400 \mathrm{~K}$ interval and (ii) a superconducting magnet up to $9 \mathrm{~T}$ (superconducting magnets up to 16 Tesla are available). The native hardware control of temperature and magnetic field provided by the PPMS was used and a new extension, permitting the collection of Raman spectroscopy data, was designed and fabricated. The Raman extension comprises the following parts: (a) The custom-made Raman top part (including several imaging lenses, beamsplitter, notch filter, white light illumination and CCD camera) and a modified PPMS Raman insert (using multifunction probe temperature sensor) serving as a sample/DAC holder. (b) A miniature nonmagnetic diamond anvil cell (DAC). (c) A $488 \mathrm{~nm}$ argonion CW laser system. (d) A Shamrock spectrometer with thermoelectrically cooled CCD detector.

The data acquisition, including Raman spectroscopic module (Solis, Andor Technology Ltd.), as well as software control of PPMS temperature and magnetic fields, was implemented using the QD PPMS third party option. A schematic view of the set-up is shown in Figure 1. It comprises a probe, akin to the commercially available multifunction probe (MFP), albeit mechanically more resistant, providing optical and electrical contacts to the sample, and an optical illumination/data collection system. A single sapphire optical window creates an interface between the evacuated interior of the magnet cryostat and the Raman top part.

Pictures of the upper optical part of the PPMS Raman extension, sample holder and diamond anvil pressure cell are shown in Figure 2(a)-(c), respectively. Magnetic field may be applied up the (+/-) maximum accessible value of the PPMS system. Electric field can also be applied, as indicated by the arrow in Figure 1, using an external voltage source. The high pressure conditions are created using miniature nonmagnetic diamond anvil cell (DAC), specially designed to hold pressure stable during temperature scans [14]. The 


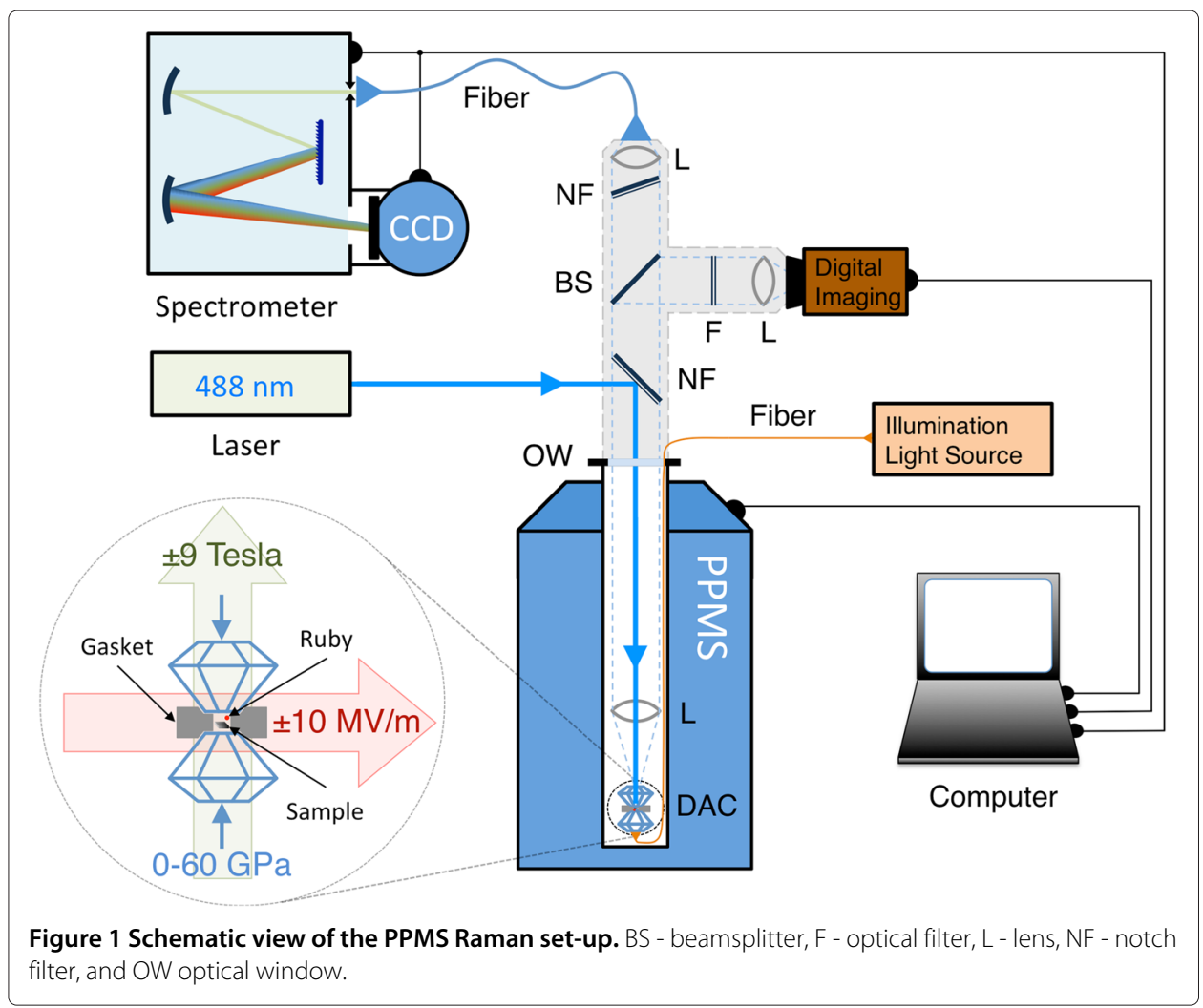

DAC is made in a piston-cylinder configuration with a vertical optical axis and a maximum diameter of 10-13 $\mathrm{mm}$. The maximal pressure for diamonds with $0.3 \mathrm{~mm}$ culets is $\sim 60 \mathrm{GPa}$. Our Raman extension is suitable for long-term experiments lasting several days, exploiting the typical operational parameter space of the PPMS.

\section{Optical design}

Raman spectra are recorded with a SR-303I-A Shamrock 303i spectrometer (Andor Technology) coupled by a single mode optical fiber to the PPMS Raman extension. The spectrometer is equipped with $1200 \mathrm{l} / \mathrm{mm}$ grating and thermoelectrically cooled multichannel CCD detector (iDus, Andor Technology, $1024 \times 256$ pixels, $-50^{\circ} \mathrm{C}$ ). A linearly polarized argon ion laser ( $488 \mathrm{~nm}$ line) is used for the excitation at powers up to $15 \mathrm{~mW}$. The spectral axis is calibrated by the fluorescence lines of a neon lamp. Inside the PPMS, Raman spectra are collected in the back scattering geometry, at a resolution of about 4 $\mathrm{cm}^{-1}$. Elastically backscattered fundamental light $(488 \mathrm{~nm})$ is filtered out using a narrow band notch filter (Semrock Inc.). The accuracy of the spectral measurements, resulting from the wavelength calibration procedure and experimental conditions, is estimated to be about $2 \mathrm{~cm}^{-1}$. Typical acquisition times vary between $20-120 \mathrm{sec}$. Experimentally accessible ranges of temperature, pressure, magnetic and electric fields available for spectroscopic acquisitions correspond to 3 - $350 \mathrm{~K}$ (higher temperatures with additional heating possible), 0 - $60 \mathrm{GPa}, 0$ - \pm 9 Tesla and 0 - $\pm 10 \mathrm{MV} / \mathrm{m}$, respectively. So far, spectral acquisitions involving strong electric fields have been carried out outside the PPMS only. Typically, the experimental arrangement involves application of hundreds of volts across a sample which is few tens of micrometers thick. 


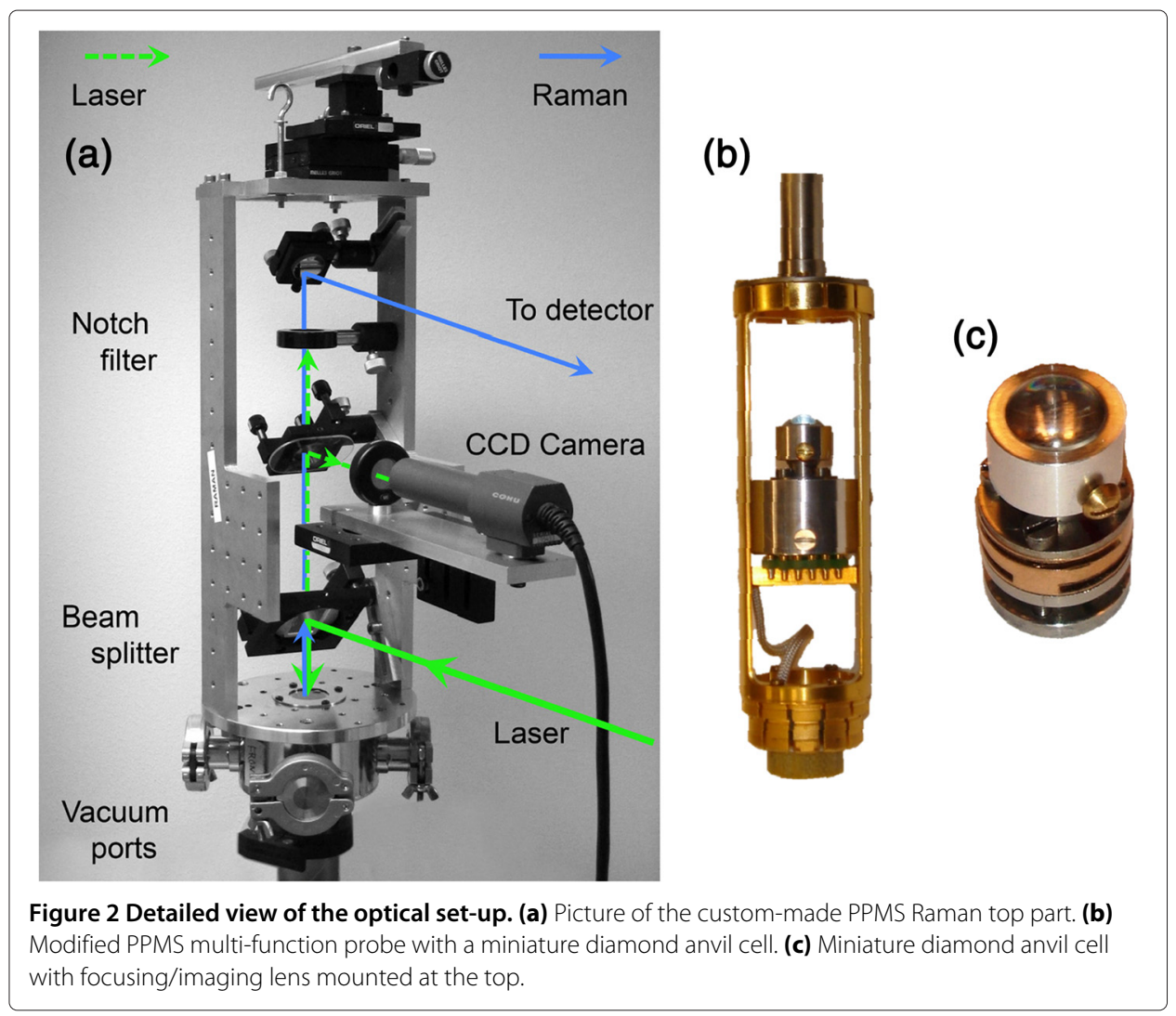

\section{Alignment and measurement routine}

Measurements are taken in the back scattering geometry. Due to space limitation inside the cryostat and low temperature conditions right angle scattering geometry is difficult to achieve in the described set-up. For sample illumination and focusing, white light is coupled via an optical fiber into the set-up and applied in transmission. Prior the measurements the optical system is pre-aligned and tested outside the cryostat using a room-temperature probe stand.

A standard (high-pressure) experiment is done in the following way. (i) The sample is mounted onto the Raman probe using ambient-pressure sample holder or DAC. For highpressure experiments specific sample-, ruby- and pressure transmitting medium loading procedures have to be applied as described in Ref. [14]. (ii) The sample is aligned with the optical set-up and inserted into the PPMS cryostat. (iii) Single measurements are performed manually or an automatized measurement sequence is programmed using the PPMS third-party option together with the spectrometer software scripting option.

\section{Exemplary results and discussion}

Ruby fluorescence lines

$\mathrm{Cr}^{3+}$-doped $\mathrm{Al}_{2} \mathrm{O}_{3}$, also known as ruby, is a well-established standard material with interesting properties that led to significant technological advances both in academia and industry [15]. For instance, ruby was the material that led to the invention of the first laser by Maiman in 1960 [16]. The characteristic red color of ruby is related to electronic transitions involving the $\mathrm{Cr}^{3+}$ ions manifested in the two fluorescence lines, $\mathrm{R} 1$ and $\mathrm{R} 2$ [17]. 
Ruby is furthermore a well-established pressure gauge in optical high-pressure experiments [18] and hence has been studied extensively. Zeeman splitting in single crystalline ruby has been observed in pulsed magnetic fields up to $60 \mathrm{~T}$ and hydrostatic pressure up to $10 \mathrm{GPa}$ [19]. In the presented PPMS Raman set-up static magnetic fields up to $9 \mathrm{~T}$ (16 T with different cryostat) and hydrostatic pressure up to $60 \mathrm{GPa}$ can be achieved. In Figure 3 the magnetic field dependence for the ruby $\mathrm{R} 1$ line at $5 \mathrm{~K}$ and $34 \mathrm{GPa}$ is depicted. Ruby can hence serve as a local (in-situ) probe of the magnetic field $(\Delta \lambda / \Delta H \sim 0.046 \mathrm{~nm} / \mathrm{T})$.

\section{Hydrogen conversion}

Hydrogen is the most common element in the known universe. It exists in two different spin isomers, ortho hydrogen with two parallel proton spins and para hydrogen with two antiparallel proton spins. At room temperature and ambient pressure the ortho- to para hydrogen ratio is approximately 1:3. This equilibrium ratio is temperature dependent and at low temperatures the equilibrium state is para hydrogen. $\mathrm{H}_{2}$ conversion, from orthoto para hydrogen can be traced by measuring $\mathrm{H}_{2}$ rotational modes (rotons), i.e. the integrated intensities of the rotational Raman peaks. Figure 4(a) shows $\mathrm{H}_{2}$ conversion data collected using the PPMS Raman extension. The $\mathrm{H}_{2}$ conversion rate is strongly enhanced under hydrostatic pressure [20]. In the presented PPMS Raman set-up the combined effect of hydrostatic pressure and high magnetic fields can be studied. The evolution of the rotational Raman modes (from $0 \mathrm{~h}$ (black line) to $8 \mathrm{~h}$ ) at $\sim 20 \mathrm{~K}, 9 \mathrm{~T}$ magnetic field, and 27 GPa hydrostatic pressure is depicted. Vibrational Raman modes (vibrons) of hydrogen can be observed in a factor of 10 higher frequency range. Figure 4(b) illustrates the hydrogen $\mathrm{Q}_{1}(1)$ vibron at temperatures close to room temperature $(300 \mathrm{~K})$ and at low temperature $(5 \mathrm{~K})$ for both zero and $9 \mathrm{~T}$ magnetic fields. Hydrogen vibron modes are sensitive to structural changes and a clear difference between fluid and solid hydrogen is observed.

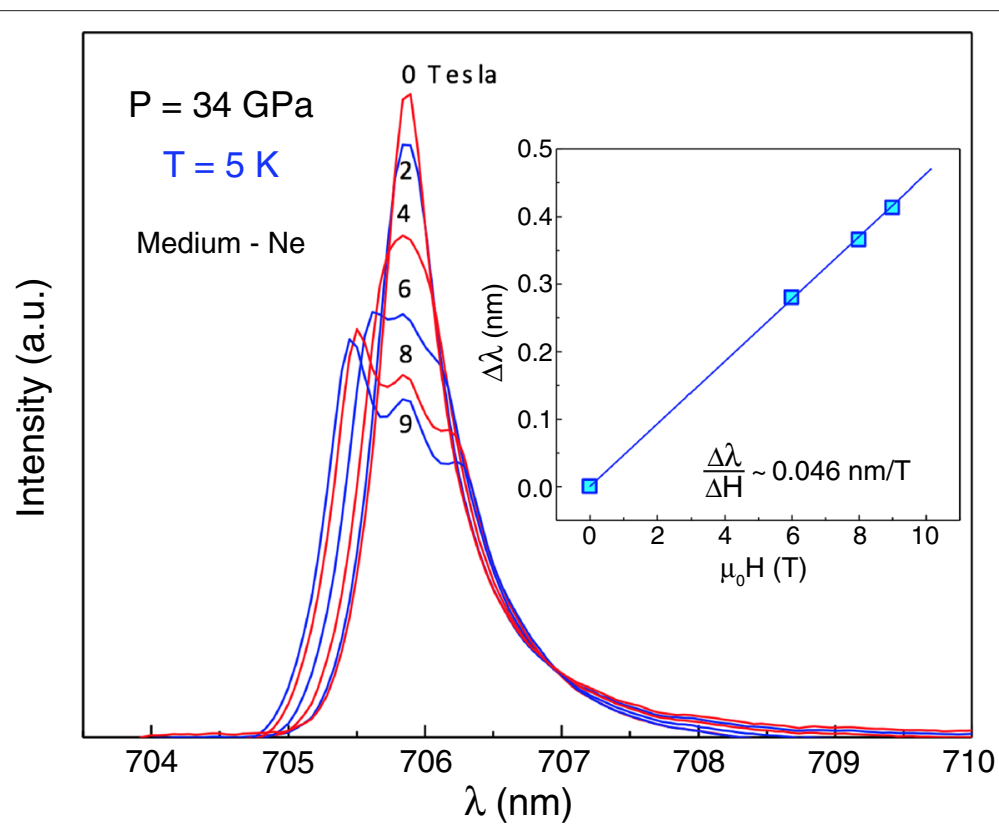

Figure 3 Exemplary measurements showing the magnetic field dependence of ruby $\left(\mathrm{Cr}^{3+}\right.$-doped $\mathrm{Al}_{2} \mathrm{O}_{3}$ ) fluorescence lines at $5 \mathrm{~K}$ and $34 \mathrm{GPa}$. Inset shows the shift of ruby fluorescence line with applied magnetic field. 

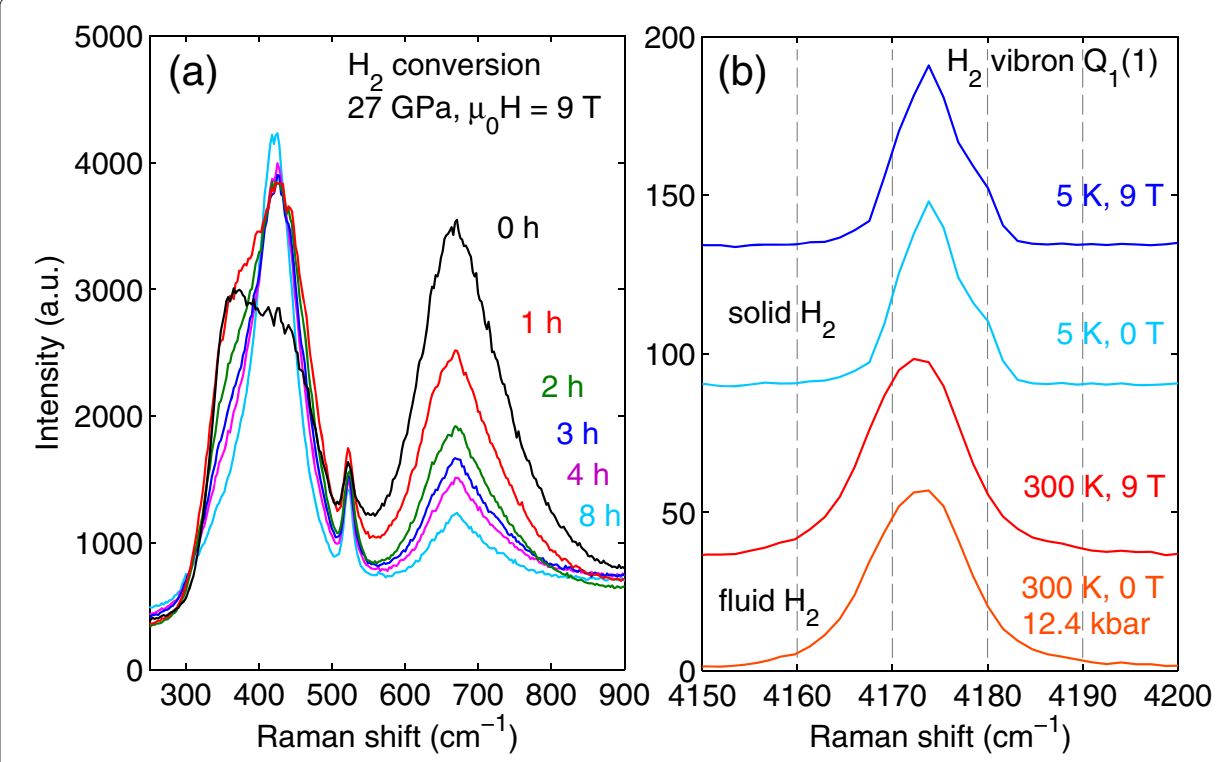

Figure 4 Exemplary measurements showing (a) the ortho-to-para conversion of molecular hydrogen at $\sim 20 \mathrm{~K}$ at applied magnetic field $(9 \mathrm{~T})$ and high pressure $(27 \mathrm{GPa})$ and $(\mathrm{b})$ the hydrogen vibron $\mathrm{Q}_{1}(1)$ at $5 \mathrm{~K}$ and $300 \mathrm{~K}$ in zero and $9 \mathrm{~T}$ magnetic field.

\section{Spin-phonon coupling in a strongly correlated oxide}

Langasite materials are well known for their piezoelectric and non-linear optical properties [21,22]. A recent interest in the magnetic and electrical properties of langasite materials is due to the discovery that they could crystalize in a Kagomé-like lattice structure consisting of rare-earth and transition metal cations [23,24]. While a detailed report of the study of the spin-phonon coupling in those materials is beyond the scope of this article, we present here Raman spectroscopy data collected in our set-up as a function of temperature and magnetic fields. Simple qualitative analysis are also included, in order to illustrate the typical studies which may be performed on such materials.

$\mathrm{Ba}_{3} \mathrm{NbFe}_{3} \mathrm{Si}_{2} \mathrm{O}_{14}$ crystallizes in the non-centrosymmetric trigonal P321 space group with 23 unit cell atoms. Magnetic $\mathrm{Fe}^{3+}$ ions are arranged in triangle units on a triangular lattice [23]. The structure is non-polar and hence no spontaneous polarization can be expected for point group 32 [25]. It has been reported that $\mathrm{Ba}_{3} \mathrm{NbFe}_{3} \mathrm{Si}_{2} \mathrm{O}_{14}$ undergoes an antiferromagnetic phase transition at approximately $27 \mathrm{~K}$ [23]. A much larger CurieWeiss temperature of $\theta \approx-175 \mathrm{~K}$ [26], reflects the large magnetic frustration in the system [27]. The magnetic transition is accompanied by a symmetry lowering from P321 to C2 [28]. Further details on the magnetic structure and Raman mode assignment can be found in literature $[25,29,30]$.

Unpolarized Raman spectra on a single crystal of $\mathrm{Ba}_{3} \mathrm{NbFe}_{3} \mathrm{Si}_{2} \mathrm{O}_{14}$ recorded as a function of temperature and magnetic field are shown in Figure 5. All $T$ and $H$ dependent measurements were done with an the incident beam along the [1-10] direction. For better visualization spectra $(50-300 \mathrm{~K})$ have been shifted vertically with a linear offset. The phonon spectrum of $\mathrm{Ba}_{3} \mathrm{NbFe}_{3} \mathrm{Si}_{2} \mathrm{O}_{14}$ exhibits a frequency hardening and narrowing of the line-width on reducing temperature.

In a quasi-harmonic approximation, the frequency shift can be attributed to the volume change only and anharmonic effects due to phonon-phonon and spin-phonon 


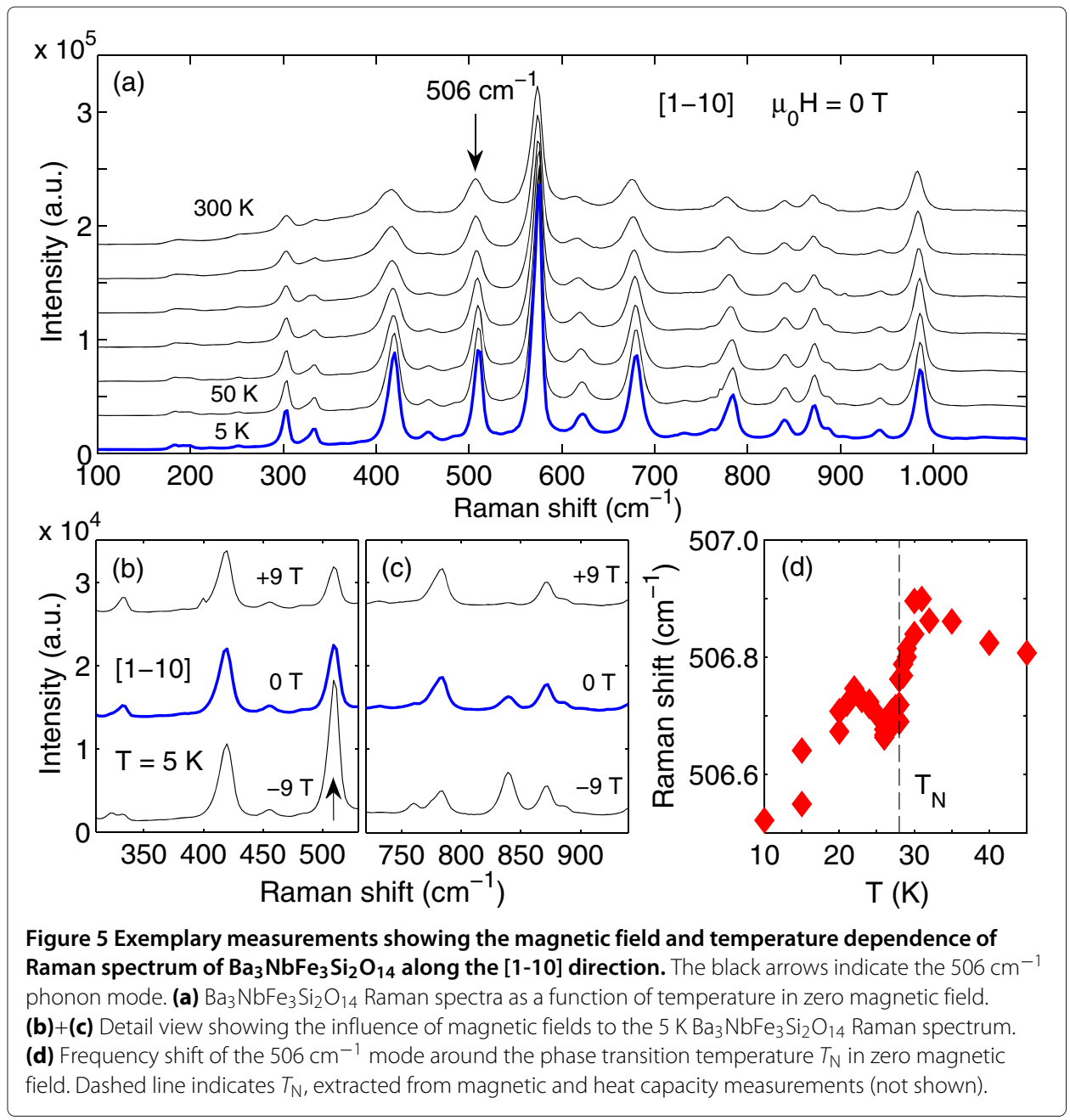

interaction are ignored. Nevertheless, additional changes in the phonon frequencies when approaching $T_{\mathrm{N}}$ are evident, as shown in Figure 5(d). For instance, the $506 \mathrm{~cm}^{-1}$ phonon mode reveals softening behavior with an onset above $T_{\mathrm{N}}$ and a subsequent hardening below $25 \mathrm{~K}$. Similar behavior is observed for the other modes, suggesting spinphonon coupling. Figure 5(d) also illustrates precision and stability of the Raman set-up, which allow detection of a magnetic transition involving a change of only a fraction of wavenumber.

The magnetic field dependence of the unpolarized Raman spectra of $\mathrm{Ba}_{3} \mathrm{NbFe}_{3} \mathrm{Si}_{2} \mathrm{O}_{14}$ at $5 \mathrm{~K}$ is shown in Figure $5(\mathrm{~b})+(\mathrm{c})$. In this measurement the magnetic field was set to the highest value $(9 \mathrm{~T})$ and the first Raman spectrum was collected. Subsequently, the magnetic field was swept from $9 \mathrm{~T}$ to $-9 \mathrm{~T}$. At a first glance, a comparably 'strong' change in the integrated intensity of the 310,416 and $506 \mathrm{~cm}^{-1}$ modes and in the position of the $615 \mathrm{~cm}^{-1}$ mode are found. Furthermore, several new modes appear at 250, 322, 760 and $840 \mathrm{~cm}^{-1}$ when the field is changed from $9 \mathrm{~T}$ to $-9 \mathrm{~T}$. The influence of the magnetic field on phonon modes 506, 573, 675 and $982 \mathrm{~cm}^{-1}$ is weak and only the 416 and $615 \mathrm{~cm}^{-1}$ modes exhibit major changes in the frequency [31]. 
Recently, the magnetic (and structural) properties of related langasites were found to be greatly affected by application of pressure [32], suggesting the relevance of T,P,Hdependent Raman studies on $\mathrm{Ba}_{3} \mathrm{NbFe}_{3} \mathrm{Si}_{2} \mathrm{O}_{14}$ and other strongly correlated materials.

\section{Conclusion}

A Raman and luminescence spectroscopy system for the study of strongly correlated electron materials is presented. The system is designed as an extension of the Quantum Design, Inc. PPMS and allows for measurements in a large temperature range (3 - $350 \mathrm{~K})$, high magnetic fields $(0-9 \mathrm{~T})$, high pressure $(0-60 \mathrm{GPa})$ and electric field strengths up to $10 \mathrm{MV} / \mathrm{m}$. Gaseous, liquid and solid materials, in amorphous, poly- and single-crystalline bulk form or as a thin film can be studied. Exemplary results for ruby and solid hydrogen at low temperature and high hydrostatic pressure and high magnetic field are shown. Finally results of the study of the spin-phonon coupling in the complex transition metal oxide $\mathrm{Ba}_{3} \mathrm{NbFe}_{3} \mathrm{Si}_{2} \mathrm{O}_{14}$ are presented and discussed.

Competing interests

The authors declare that they have no competing interests.

\section{Authors' contributions}

$\mathrm{MH}$ participated in building the experimental apparatus, performed experimental studies of ruby, $\mathrm{H}_{2}$, and $\mathrm{Ba}_{3} \mathrm{NbFe}_{3} \mathrm{Si}_{2} \mathrm{O}_{14}$ and drafted the manuscript. PL initiated the study and participated in design and building the experimental apparatus and performed experimental studies of ruby and $\mathrm{H}_{2}$. RM participated in design of the study on strongly correlated materials and helped to draft the manuscript. AG participated in experimental studies of ruby. VS participated experimental studies of ruby and $\mathrm{H}_{2}$ and helped to draft the manuscript. All authors read and approved the final manuscript.

\section{Acknowledgements \\ We are very grateful to Dr. Y. Tokunaga, and Profs Y. Taguchi and Y. Tokura for advices and support in the floating zone growth of $\mathrm{Ba}_{3} \mathrm{NbFe}_{3} \mathrm{Si}_{2} \mathrm{O}_{14}$ single-crystal. Financial support from the Carl Tryggers Stiftelse för Vetenskaplig Forskning (project CTS 09:217), the Swedish Foundation for International Cooperation in Research and Higher Education (STINT), the Swedish Research Council (VR) and the Göran Gustafsson Foundation is acknowledged. MH thanks the Swedish Research Council (VR, contract 2012-6562) for financial support and Profs M. Fiebig and O. Tjernberg for hosting him during his postdoc. AG thanks the Russian Foundation for Basic Researches (grant 14-02-00483-a), and the program of RAS "Physics of elementary particles, fundamental nuclear physics and nuclear technologies" for financial support.}

\footnotetext{
Author details

${ }^{1}$ KTH Royal Institute of Technology, ICT Materials Physics, Electrum 229, SE-164 40 Kista, Sweden. ${ }^{2}$ Department of Materials, ETH Zürich, Vladimir-Prelog-Weg 4, CH-8093 Zürich, Switzerland. ${ }^{3}$ Department of Earth Sciences, Uppsala University, Villavägen 16, SE-752 36 Uppsala, Sweden. ${ }^{4}$ Department of Engineering Sciences, Uppsala University, Box 534, SE-751 21 Uppsala, Sweden. ${ }^{5}$ Institute of Crystallography, Russian Academy of Sciences, Leninsky pr. 59, 119333 Moscow, Russia. ${ }^{6}$ Institute for Nuclear Research, Russian Academy of Sciences, 60-letiya Oktyabrya prospekt 7a, 117312 Moscow, Russia. ${ }^{7}$ Geophysical Laboratory, Carnegie Institution of Washington, 5251 Broad Branch Road NW, 20015 Washington DC, USA.
}

Received: 3 October 2014 Accepted: 9 February 2015

Published online: 27 February 2015

\section{References}

1. Bibes M, Barthelemy A. Oxide Spintronics. IEEE Trans Elec Dev. 2007:54(5):1003.

2. Scott JF. Applications of magnetoelectrics. J Mater Chem. 2012;22:4567.

3. Choudhury D, Mandal P, Mathieu R, Hazarika A, Rajan S, Sundaresan A, et al. Near-Room-Temperature Colossal Magnetodielectricity and Multiglass Properties in Partially Disordered La $2 \mathrm{NiMnO}_{6}$. Phys Rev Lett. 2012;108:127201.

4. Grinberg I, West DV, Torres M, Gou G, Stein DM, Wu L, et al. Perovskite oxides for visible-light-absorbing ferroelectric and photovoltaic materials. Nature. 2013;503(7477):509-12.

5. Hosono H. Exploring electro-active functionality of transparent oxide materials. Jpn J Appl Phys. 2013;52:090001.

6. Tokura Y, Nagaosa N. Orbital Physics in Transition-Metal Oxides. Science. 2000;288:462.

7. Devereaux TP, Hackl R. Inelastic light scattering from correlated electrons. Rev Mod Phys. 2007;79:175-233.

8. Guennou M, Bouvier P, Chen GS, Dkhil B, Haumont R, Garbarino G, et al. Multiple high-pressure phase transitions in $\mathrm{BiFeO}_{3}$. Phys Rev B. 2011;84:174107.

9. Iliev MN, Gospodinov MM, Singh MP, Meen J, Truong KD, Fournier P, et al. Growth, magnetic properties, and Raman scattering of $\mathrm{La}_{2} \mathrm{NiMnO}_{6}$ single crystals. J Appl Phys. 2009;106(2):023515.

10. Quantum Design Inc. Physical Property Measurement System (PPMS). http://www.qdusa.com/products/ppms.html 2015 Accessed 6 Feb 2015.

11. Hudl M, Mathieu R, Ivanov SA, Weil M, Carolus V, Lottermoser T, et al. Complex magnetism and magnetic-field-driven electrical polarization of $\mathrm{CO}_{3} \mathrm{TeO}_{6}$. Phys Rev B. 2011;84:180404. 
12. Isaacs ED, Heiman D. Fiber optics for Raman scattering at low temperatures and high-magnetic fields. Rev Sci Instrum. 1987;58(9):1672-4.

13. Matsuda $Y$, Kuroda N, Nishina Y. High-field, high-pressure, and low-temperature magneto-optical apparatus using a diamond anvil cell. Rev Sci Instrum. 1992;63(12):5764-6.

14. Gavriliuk AG, Mironovich AA, Struzhkin W. Miniature diamond anvil cell for broad range of high pressure measurements. Rev. Sci. Instrum. 2009;80:043906.

15. Syassen K. Ruby under pressure. High Pressure Res. 2008;28(2):75-126.

16. Maiman TH. Stimulated Optical Radiation in Ruby. Nature. 1960;187:493-94.

17. García-Lastra JM, Barriuso MT, Aramburu JA, Moreno M. Origin of the different color of ruby and emerald. Phys. Rev. B. 2005;72:113104.

18. Mao HK, Xu J, Bell PM. Calibration of the Ruby pressure gauge to 800 kBar under quasi-hydrostatic conditions. J Geophys Res-Solid. 1986;91(B5):4673-6.

19. Millot M, Broto J-M, Gonzalez J. High-field Zeeman and Paschen-Back effects at high pressure in oriented ruby. Phys Rev B. 2008;78:155125.

20. Eggert JH, Karmon E, Hemley RJ, Mao HK, Goncharov AF. Pressure-enhanced ortho-para conversion in solid hydrogen up to 58 GPa. Proc Nat Acad Sci USA. 1999;96(22):12269-72.

21. Kaminskii AA, Mill BV, Khodzhabagyan GG, Konstantinova AF, Okorochkov Al, Silvestrova IM. Investigation of trigonal $\left(\mathrm{La}_{1-x} \mathrm{Nd}_{x}\right)_{3} \mathrm{Ga}_{5} \mathrm{SiO}_{14}$ Crystals. Phys Stat Sol (A). 1983;80:387.

22. Sato J, Takeda H, Morikoshi H, Shimamura K, Rudolph P, Fukuda T. Czochralski growth of $\mathrm{RE}_{3} \mathrm{Ga}_{5} \mathrm{SiO}_{14}(\mathrm{RE}=\mathrm{La}$, $\operatorname{Pr}$ $\mathrm{Nd}$ ) single crystals for the analysis of the influence of rare earth substitution on piezoelectricity. J Cryst Growth. 1998;191:746.

23. Marty K, Simonet V, Ressouche E, Ballou R, Lejay P, Bordet P. Single domain magnetic helicity and triangular chirality in structurally enantiopure $\mathrm{Ba}_{3} \mathrm{NbFe}_{3} \mathrm{Si}_{2} \mathrm{O}_{14}$. Phys Rev Lett. 2008;101(24):247201.

24. Zhou HD, Lumata LL, Kuhns PL, Reyes AP, Choi ES, Dalal NS, et al. $\mathrm{Ba}_{3} \mathrm{NbFe}_{3} \mathrm{Si}_{2} \mathrm{O}_{14}$ : A New Multiferroic with a $2 \mathrm{D}$ Triangular Fe $\mathrm{F}^{3+}$ Motif. Chem Mater. 2009;21(1):156-9.

25. Birss RR. Symmetry and Magnetism: North-Holland Publishing Company - Amsterdam; 1966.

26. Marty K, Bordet $\mathrm{P}$, Simonet $\mathrm{V}$, Loire M, Ballou R, Darie C, et al. Magnetic and dielectric properties in the langasite-type compounds: A(3)BFe(3)D(2)O(14) (A=Ba, Sr, Ca; B = Ta, Nb, Sb; D = Ge, Si). Phys Rev B. 2010;81: 054416.

27. Greedan JE. Geometrically frustrated magnetic materials. J Mater Chem. 2001;11:37-53.

28. Lyubutin IS, Naumov PG, Mill' BV, Frolov KV, Demikhov El. Structural and magnetic properties of the iron-containing langasite family $A_{3} M \mathrm{Fe}_{3} X_{2} \mathrm{O}_{14}(A=\mathrm{Ba}, \mathrm{Sr} ; M=\mathrm{Sb}, \mathrm{Nb}, \mathrm{Ta} ; X=\mathrm{Si}$, Ge) observed by Mössbauer spectroscopy. Phys Rev B. 2011;84:214425.

29. Li Y, Lu G, Yang H, Lan J, Zhang J, Huang W, et al. Lattice vibration of $\mathrm{Sr}_{3} \mathrm{TaGa}_{3} \mathrm{Si}_{2} \mathrm{O}_{14}$ single crystal. Phys Stat Solidi (b). 2007;244(2):518-28.

30. Kroumova E, Aroyo MI, Perez-Mato JM, Kirov A, Capillas C, Ivantchev S, et al. Bilbao crystallographic server: Useful databases and tools for phase-transition studies. Phase Transit. 2003;76(1-2):155-70.

31. Hudl M. Magnetic materials with tunable thermal, electrical, and dynamic properties: An experimental study of magnetocaloric, multiferroic, and spin-glass materials: Uppsala: Uppsala Dissertations from the Faculty of Science and Technology 98; 2012.

32. Gavriliuk AG, Lyubutin IS, Starchikov SS, Mironovich AA, Ovchinnikov SG, Trojan IA, et al. The magnetic P-T phase diagram of langasite $\mathrm{Ba}_{3} \mathrm{TaFe}_{3} \mathrm{Si}_{2} \mathrm{O}_{14}$ at high hydrostatic pressures up to $38 \mathrm{GPa}$. Appl Phys Lett. 2013;103(16):

\section{Submit your manuscript to a SpringerOpen ${ }^{\circ}$ journal and benefit from:}

- Convenient online submission

- Rigorous peer review

- Immediate publication on acceptance

- Open access: articles freely available online

- High visibility within the field

- Retaining the copyright to your article

Submit your next manuscript at $\gg$ springeropen.com 